\title{
The Neuropsychological Syndrome of Agenesis of the Corpus Callosum
}

\author{
Warren S. Brown ${ }^{1}$ and Lynn K. Paul ${ }^{2}$ \\ ${ }^{1}$ Fuller Graduate School of Psychology, Travis Research Institute, 180 N. Oakland Ave., \\ Pasadena, CA 91101, USA \\ ${ }^{2}$ California Institute of Technology, Division of Humanities and Social Sciences, 1200 E. \\ California Blvd., Pasadena, CA 91125, USA
}

Corresponding Author:

Warren S. Brown

Fuller Graduate School of Psychology

180 N. Oakland Ave.

Pasadena, CA 91101

Email: wsbrown@,fuller.edu 626-584-5525-office

Keywords: corpus callosum, agenesis of the corpus callosum

Word Count: Abstract (150); Article (2694) 


\begin{abstract}
Background: Agenesis of the corpus callosum $(\mathrm{AgCC})$ involves congenital absence of all or part of the corpus callosum. Because the disorder can only be firmly diagnosed via neuroradiology, it has a short research history, and only recently has the cognitive syndrome become clear. Purpose: Our purpose is to review the primary deficits in AgCC that constitute the core syndrome. Conclusions: The cores syndrome includes: (1) Reduced interhemispheric transfer of sensory-motor information; (2) Reduced cognitive processing speed; (3) Deficits in complex reasoning and novel problem-solving. These domains do not appear to reflect different neuroanatomical abnormalities, but rather different domains of expression of reduced interhemispheric communication from callosal absence. Implications: These core deficits are expressed across various domains of cognitive, behavioral, and social functioning. The impact of these deficits varies across development and may be moderated by individual factors such as cooccurrence of other neurodevelopmental conditions, general intellectual capacity, and environmental support.
\end{abstract}




\section{Introduction}

This paper describes what we believe to be the core syndrome of agenesis of the corpus callosum (AgCC) as displayed in adults who have few, if any, other neurological abnormalities, and have grossly intact general intelligence. Although $\mathrm{AgCC}$ was previously thought to be exceedingly rare, increased clinical use of neuroimaging has resulted in higher detection rates in relatively normally functioning individuals. Studies conducted with this larger pool of patients are providing greater understanding of the role of the corpus callosum in cognition and behavior. The accumulating data also provides families and clinicians with more nuanced insights into the patterns of cognitive capacities that influence learning, daily behaviors, and developmental progression of individuals with $\mathrm{AgCC}$.

$\mathrm{AgCC}$ is a congenital brain malformation defined by anatomy (complete or partial absence of the corpus callosum), and not defined by behavior abnormalities (as in autism). AgCC occurs due to disruption of neural development during the 7th to 20th embryonic weeks (Edwards, Sherr, Barkovich, \& Richards, 2014). The most recent evidence indicates that AgCC occurs in at least 1 in 4,000 births, making it one of the more commonly occurring congenital brain disorders (Glass, Shaw, Ma, \& Sherr, 2008; Guillem, Fabre, Cans, Robert-Gnansia, \& Jouk, 2003; Wang, Huang, \& Yeh, 2004).

$\mathrm{AgCC}$ is often associated with a broader syndrome of brain malformation related to known toxic-metabolic conditions or genetic causes, but in $55-70 \%$ of $\mathrm{AgCC}$ cases the cause is unknown (Bedeschi et al., 2006; Schell-Apacik et al., 2008; Tang et al., 2009). When part of a broader neurodevelopmental syndrome or accompanied by other congenital brain malformations, the cognitive and behavioral impact of these other conditions would likely obscure the moderate to mild deficiencies directly related to callosal absence. In order to identify the specific 
contributions of the corpus callosum to higher cognitive abilities, this review describes the $\mathrm{AgCC}$ syndrome as it appears in individuals without any (or only very minor) other brain or body dysmorphology. These individuals typically have normal-range IQs and appear neurologically "asymptomatic" (i.e., lack of symptoms apparent in diagnostic procedures typically used in clinical neurology). In most of these individuals AgCC is discovered through routine prenatal sonogram or neuroimaging motivated by unrelated issues. In these cases, AgCC is likely to be the primary contributor to the cognitive outcome, and thus we refer to these individuals as having "Primary AgCC".

Although they have a common primary neurological finding, individuals with Primary $\mathrm{AgCC}$ are somewhat heterogeneous with respect to other minor structural brain abnormalities, some of which appear to be secondary to the callosal dysgenesis (e.g. colpocephaly, Probst Bundles) and some of which may or may not be related to the callosum (e.g. minor areas of heterotopia, interhemispheric cysts, interhemispheric lipoma). The group also varies with regard to amount of residual callosal connection (complete vs. partial), and most certainly varies at the molecular and synaptic level. To minimize the negative influence of concomitant neurological abnormalities, this review focuses on individuals with a full scale IQ above 80 . Heterogeneity notwithstanding, we present what we believe to be the core syndrome which results in the mild to moderate cognitive and psychosocial deficits in Primary AgCC.

Finally, since the corpus callosum in neurotypical children is undergoing significant myelinization and functional development into the teenage years (Giedd et al., 1994; Yakovlev, Lecours, \& Minkovski, 1967), the neuropsychological and psychosocial outcomes of AgCC seem to 'emerge' as their peers increase reliance on callosal connectivity. Thus, our commentary focuses on studies of older adolescents and adults. 


\section{The Core Syndrome of Primary AgCC}

The aim of this paper is to briefly sketch the core syndrome of Primary AgCC (i.e., deficits specifically related to callosal absence) which in turn contributes to more specific neuropsychological and psychosocial deficits in $\mathrm{AgCC}$. The following sections will offer evidence from published literature (including much of our own research) demonstrating that Primary $\mathrm{AgCC}$ is associated with a core syndrome involving:

(1) Reduced interhemispheric transfer of sensory-motor information;

(2) Increased cognitive processing time;

(3) Deficient processing of complex information and unfamiliar tasks: amplified vulnerability to increases in cognitive demands.

These three domains of dysfunction are not independent. Reduced interhemispheric interactions likely contribute to slower processing time and difficulty in complex problemsolving, which are themselves inter-related. These core deficits contribute to many other specific deficiencies we describe briefly (see figure 1).

\section{Diminished Interhemispheric Integration of Sensory-Motor Information}

The current understanding of callosal function came primarily from studying individuals who had a surgical commissurotomy - severing of all of the cerebral commissures as a treatment for epilepsy. Commissurotomy and $\mathrm{AgCC}$ both result in a lack of callosal connections between hemispheres, but they are distinguished by (1) the presence of the other cerebral commissures in most cases of $\mathrm{AgCC}$ (e.g., anterior commissure is present in all cases of AgCC we describe), and (2) by the point in development at which the hemispheres are disconnected. Early investigations of interhemispheric transfer and integration of sensory and motor information in $\mathrm{AgCC}$ discovered that congenital absence of callosal connections does not cause 
a full "disconnection syndrome" as is seen following commissurotomy (Sperry, 1968; Bogen \& Frederiks, 1985; Sperry, Gazzaniga, Bogen, Vinken, \& Bruyn, 1969).

Unlike commissurotomy, limitations of interhemispheric transfer in $\mathrm{AgCC}$ are contingent upon the complexity of information being transferred. Numerous studies have shown that individuals with $\mathrm{AgCC}$ are capable of interhemispheric integration of easily encoded visual and tactile information (e.g., Brown, Jeeves, Dietrich, \& Burnison, 1999; Chiarello, 1980; Jeeves \& Ettlinger, 1965; Lassonde, Sauerwein, Chicoine, \& Geoffroy, 1991; Saul \& Sperry, 1968). However, diminished interhemispheric transfer in $\mathrm{AgCC}$ is evident in studies requiring transfer of more complex (and less familiar) information (e.g., Brown et al., 1999; Bryden \& Zurif, 1970; Buchanan, Waterhouse, \& West Jr., 1980; Geffen, Nilsson, Quinn, \& Teng, 1985; Jeeves, 1979).

A study by Brown et al. (1999) illustrates key differences in interhemispheric transfer of sensory information in individuals with $\mathrm{AgCC}$ and individuals with commissurotomy through the use of two tachistoscopic bilateral visual field matching tasks (letters and dot-patterns). As predicted by studies of the 'disconnection syndrome', the two commissurotomy patients in this study could not match bilateral presentations of either letters or dot-patterns above the level of chance. In contrast, participants with $\mathrm{AgCC}$ performed as well as neurotypical controls when matching two bilaterally and simultaneously flashed letters, with their limitations in interhemispheric transfer only becoming evident in impaired bilateral visual field matching of the less familiar and easily encoded random dot patterns.

The mediating effect of encoding complexity on interhemispheric transfer in $\mathrm{AgCC}$ has also been demonstrated in studies of tactual-spatial information transfer using the Tactual Performance Test (Dunn, Paul, Schieffer, \& Brown, 2000; Sauerwein \& Lassonde, 1994; Sauerwein, Lassonde, Cardu, \& Geoffroy, 1981), Finger Localization Test (Dunn et al., 2000; Geffen et al., 1985; 
Sauerwein \& Lassonde, 1994) and tactual recognition (Jeeves \& Silver, 1988). As with sensory transfer, individuals with AgCC exhibited intact performance at the lowest levels of sensory-motor difficulty, with significant declines in performance occurring as tasks requiring transfer of more complex (less easily encoded) information.

Finally, limitations in interhemispheric transmission also interfere with fine motor coordination of the two hands in individuals with AgCC (Jeeves, Silver, \& Jacobson, 1988; Jeeves, Silver, \& Milner, 1988), as well as in individuals with surgical transection of either the anterior or posterior callosum (Eliassen, Baynes, \& Gazzaniga, 2000; Preilowski, 1972). Using the Bimanual Coordination Test (BCT) - a test based on an Etch-a-Sketch toy (Brown, 1991) we found that bimanual motor coordination was slower and less accurate in adults with AgCC than in neurotypical adults (Mueller, Marion, Paul, \& Brown, 2009), but performance of adults with AgCC was similar to that of neurotypical children for whom the corpus callosum was not yet fully developed (Marion, Kilian, Naramor, \& Brown, 2003). Notably, even in neurotypical adults, poor structural integrity of motor connections via the corpus callosum becomes more strongly associated with poor bimanual coordination performance as task complexity increases (Gooijers, et al, 2013).

\section{Reduced Speed of Cognitive Processing}

Speed is a fundamental feature of all cognitive processes. Consequently, slow processing speed may interfere with abilities in multiple domains. Processing speed is highly vulnerable to disruptions in white matter connectivity, particularly the CC (Solmaz et al., 2017; Ubukata et al., 2016; Kourtidou, et al, 2012; Mathias, et al., 2004). Studies described in the previous section offer clear evidence of slowed sensory and motor reaction times in individuals with AgCC. Slow processing speed is also evident in other cognitive tests. For example, in a sample of 32 adults 
with complete AgCC, we found that WAIS-III processing speed index scores were significantly lower on average than verbal, perceptual, and working memory indices (Erickson, Young, Paul, \& Brown, 2013).

Slow processing speed was also implicated in a study of cognitive inhibition in adults with AgCC. On the Color-Word subtest of the Delis-Kaplan Executive Function System, we found deficient cognitive inhibition and flexibility in adults with AgCC compared to age- and IQmatched controls, but regression analyses indicated that these differences in cognitive control were primarily the consequence of slowed processing speed (Marco et al., 2012). Thus, processing speed limitations may have wide-ranging implications for cognition and behavior in AgCC. However, as found in studies of callosal damage in traumatic brain injury, impairments in processing speed are exacerbated on tasks with greater information processing demands (Mathias, et al, 2004).

\section{Difficulty with Complex Processing}

Psychometric research in neurotypical adults has demonstrated that interhemispheric resources are recruited to assist with cognitively complex tasks (Koivisto, 2000; Reuter-Lorenz et al., 1999; Weissman \& Banich, 2000) and simple tasks that are unfamiliar / unpracticed (Norman et al, 1992). However, the benefit of interhemispheric processing decreases with practice (Cherbuin \& Brinkman, 2005; Maertens \& Pollmann, 2005; Weissman \& Compton, 2003). In keeping with this finding, adults with Primary AgCC display amplified vulnerability to increases in cognitive demands, resulting in impaired reasoning, concept formation, and novel complex problem-solving, (i.e., deficits in fluid intelligence). By contrast, they do not have deficits in overlearned cognitive processes (i.e., crystallized intelligence), as supported by relatively normal (or even elevated) performance on most verbal and spatial portions of standardized intelligence scales 
(Erickson, Young, Paul, \& Brown, 2013) and on tests of basic academic skills such as single-word reading, spelling, and math calculation (Young, Erickson, Paul, \& Brown, 2013).

Impairments in abstract reasoning (Brown \& Paul, 2000; David, Wacharasindhu, \& Lishman, 1993; Gott \& Saul, 1978), concept formation (Fischer, Ryan, \& Dobyns, 1992; Imamura, Yamadori, Shiga, Sahara, \& Abiko, 1994), problem-solving (Brown, Anderson, Symington, \& Paul, 2012; Schieffer, Paul, \& Brown, 2000), and generalization (Solursh, Margulies, Ashem, \& Stasiak, 1965) have all been observed in patients with AgCC. Such deficiencies are particularly evident as task complexity increases (Schieffer, Paul, \& Brown, 2000). For example, on the simplified (color) version of the Raven's Progressive Matrices Tests (Raven, 1960, 1965) performance of adults with AgCC was consistent with individual FullScale IQ (FSIQ) scores, but performance was impaired relative to FSIQ on the more complex Standard Progressive Matrices (Schieffer, Paul, \& Brown, 2000). The benefits of practice in Primary AgCC are supported by patterns of academic achievement in a sample of adults who performed within average range on basic mathematic calculations (a skill practiced throughout school), but exhibited significant deficits on math reasoning (Wechsler Individual Achievement Test-II; Hanna, 2018).

\section{Associated Cognitive and Psychosocial Deficiencies}

These three core cognitive deficiencies impact a wider range of cognitive and psychosocial functioning. Adults with AgCC have difficulties encoding verbal and visual information in memory and spontaneously retrieving newly learned information (Erickson et al., 2014; Paul, Erickson, Hartman, \& Brown, 2016), adequately understanding non-literal and complex language (Brown, Paul, Symington, \& Dietrich, 2005; Brown, Symington, Van LanckerSidtis, Dietrich, \& Paul, 2005; Paul, Van Lancker-Sidtis, Schieffer, Dietrich, \& Brown, 2003; 
Rehmel, Brown, \& Paul, 2016), exerting cognitive inhibition and flexibility (Marco et al., 2012), formulating strategies (Brown et al., 2012) and effectively applying imagination and creativity (Paul, Schieffer, \& Brown, 2004; Young et al., under review). In addition, these core cognitive deficits negatively impact social and emotional cognition, resulting in difficulty reasoning abstractly about emotions in social context (Anderson, Paul, \& Brown, 2017; Paul et al., 2006), expressing emotions in words (Pazienza, Paul, \& Brown, 2011), interpreting sarcasm and understanding subtle aspects of social interactions (Symington, Paul, Symington, Ono, \& Brown, 2010), recognizing emotion in faces (Bridgman et al., 2014), imagining and inferring the mental, emotional, and social functioning of others (Kang, Paul, Castelli, \& Brown, 2009; Turk, Brown, Symington, \& Paul, 2010) and awareness of functional deficits (Kaplan, Brown, Adolphs, \& Paul, 2012; Mangum, 2018; Miller, Su, Paul, \& Brown, 2018). Although they appear to be secondary products of diminished interhemispheric interactions, slowed processing time, and deficient complex problem solving, these associated cognitive and social deficits may result in functionally significant impairments in adaptive skills needed in daily life (Miller, Su, Paul, \& Brown, 2018; Mangum, 2018) and reciprocal social communication (Paul, Corsello, Kennedy, \& Adolphs, 2014).

\section{Moderating Factors}

Expression of these core deficits will vary across the lifespan, as a consequence of neuroanatomic variations and concomitant conditions, and in relation to individual traits and context. We offer brief comments on each of these influences.

Since the corpus callosum in neurotypical children is undergoing significant myelinization and functional development into the teenage years (Giedd et al., 1994; Yakovlev et al., 1967), the core deficits of AgCC described above may not become pronounced relative to 
peers prior to late childhood (Paul et al., 2007). Consistent with this, we found that processing speed and problem-solving scores were consistent with FSIQ in younger children with Primary AgCC, but fell significantly below FSIQ in an older sample (over 13yrs; Schieffer, Paul, Schilmoeller, \& Brown, 2000). Nonetheless, older children with AgCC may fall behind their peers when tasks are sufficiently complex (Garrels et al., 2001) or novel (Young et al., 2013) for their developmental level. Thus, tasks that can be mastered through practice, such as reading and arithmetic, are more likely to be impaired in children with $\mathrm{AgCC}$ than in adults. In contrast, tasks such as social interaction and complex problem solving become increasingly complex in adolescence and remain complex and somewhat novel throughout life, posing an ongoing challenge to individuals with AgCC (e.g., Kang et al., 2009; Turk et al., 2010; Mangum, 2018; Miller, Su, Paul, \& Brown, 2018).

It is reasonable to expect that the impact of $\mathrm{AgCC}$ would vary in relation to degree of callosal absence, with partial $\mathrm{AgCC}$ resulting in less severe manifestations of these deficiencies than complete AgCC. However, although most of the research cited in this paper focused on complete $\mathrm{AgCC}$, studies that included persons with partial $\mathrm{AgCC}$ found their performances were distributed among the results of individuals with complete. While the explanation for this is not yet clear, it is possible that outcomes in partial $\mathrm{AgCC}$ are impacted by individual variations in how the remaining callosal interhemispheric connections are organized (Wahl et al., 2009).

Up to $45 \%$ of individuals with $\mathrm{AgCC}$ have a known chromosomal abnormality or recognizable genetic syndrome, often resulting in additional neuropathology and/or medical conditions (i.e., not Primary ACC). The nature and severity of these additional conditions will influence expression of the core deficits from $\mathrm{AgCC}$ and at the extreme may render the core deficits functionally irrelevant in daily life. 
Finally, there are inherent variations between individuals, as well as environmental influences. For example, although general intelligence did not account for the core deficits described herein, general intelligence or specific skill sets may modulate an individual's complexity threshold and markedly impact an individuals' daily adaptive functioning.

\section{Conclusion}

Research has accumulated over the last two decades allowing for the description of a pattern of deficits characteristic of AgCC. We have argued for a core syndrome associated with callosal absence in AgCC involving reduced interhemispheric transfer of sensory-motor information, slowed cognitive processing speed, and deficits in complex reasoning and novel problem-solving. However, because these cognitive deficiencies are typically mild to moderate, they are often not easily recognized. It is our hope that better description of the cognitive and psychosocial impact of $\mathrm{AgCC}$ will increase the likelihood of a diagnostic MRI in these high-functioning cases, as well as provide more complete information and helpful guidance to patients and their families regarding the likely consequences of this congenital brain disorder.

\section{Acknowledgements}

The authors of this paper declare that they have no conflicts of interest with respect to the writing of this paper or the information contained. The writing of this paper was also not supported by grant funding. 


\section{References}

Anderson, L. B., Paul, L. K., \& Brown, W. S. (2017). Emotional intelligence in agenesis of the corpus callosum. Archives of Clinical Neuropsychology, 32(3), 267-279. doi:10.1093/arclin/acx001

Bedeschi, M. F., Bonaglia, M. C., Grasso, R., Pellegri, A., Garghentino, R. R., Battaglia, M. A., . . . Borgatti, R. (2006). Agenesis of the corpus callosum: clinical and genetic study in 63 young patients. Pediatric Neurology, 34(3), 186-193.

doi: 10.1016/j.pediatrneurol.2005.08.008

Bogen, J., \& Frederiks, J. (1985). Split-brain syndromes. Handbook of Clinical Neurology (Vol. 45, pp. 99-106). Amsterdam, Netherlands: Elsevier Science Publishing Co.

Bridgman, M. W., Brown, W. S., Spezio, M. L., Leonard, M. K., Adolphs, R., \& Paul, L. K. (2014). Facial emotion recognition in agenesis of the corpus callosum. Journal of Neurodevelopmental Disorders, 6. doi:10.1186/1866-1955-6-32

Brown, W. S. (1991). The Bimanual Coordination Test: Version 1. The Travis Research Institute Papers.

Brown, W. S., Anderson, L. B., Symington, M. F., \& Paul, L. K. (2012). Decision-making in individuals with agenesis of the corpus callosum: expectancy-valence in the Iowa Gambling Task. Archives of Clinical Neuropsychology, 27(5), 532-544. doi:10.1093/arclin/acs052

Brown, W. S., Jeeves, M. A., Dietrich, R., \& Burnison, D. S. (1999). Bilateral field advantage and evoked potential interhemispheric transmission in commissurotomy and callosal agenesis. Neuropsychologia, 37(10), 1165-1180.

Brown, W. S., \& Paul, L. K. (2000). Cognitive and psychosocial deficits in agenesis of the corpus callosum with normal intelligence. Cognitive Neuropsychiatry, 5(2), 135-157. 
Brown, W. S., Paul, L. K., Symington, M., and Dietrich, R. (2005). Comprehension of humor in primary agenesis of the corpus callosum. Neuropsychologia, 43, 906-916.

Brown, W. S., Symington, M., VanLancker, D., Dietrich, R. and Paul, L. K. (2005). Paralinguistic processing in children with callosal agenesis: Emergence of neurolinguistic deficits. Brain and Language, 93, 135-139. doi: 10.1016/j.neuropsychologia.2004.09.008

Bryden, M. P., \& Zurif, E. B. (1970). Dichotic listening performance in a case of agenesis of the corpus callosum. Neuropsychologia, 8(3), 371-377.

Buchanan, D. C., Waterhouse, G. J., \& West Jr., S. C. (1980). A proposed neurophysiological basis of alexithymia. Psychotherapy and Psychosomatics, 34, 248-255.

Cherbuin, N., \& Brinkman, C. (2005). Practice makes two hemispheres almost perfect. Cognitive Brain Research, 24(3), 413-422.

Chiarello, C. (1980). A house divided? Cognitive functioning with callosal agenesis. Brain and Language, 11, 128-158.

David, A. S., Wacharasindhu, A., \& Lishman, W. A. (1993). Severe psychiatric disturbance and abnormalities of the corpus callosum: review and case series. Journal of Neurology, Neurosurgery, and Psychiatry, 56, 85-93.

Dunn, C., Paul, L., Schieffer, B., \& Brown, W. (2000). Spatial tactile interhemispheric transfer and task complexity in agenesis of the corpus callosum [Abstract]. Presented at the Twenty-Eighth Annual International Neuropsychological Society Conference: February 912, 2000 Denver, Colorado. Journal of the International Neuropsychological Society, 6(2), 165.

Edwards, T. J., Sherr, E. H., Barkovich, A. J., \& Richards, L. J. (2014). Clinical, genetic and imaging findings identify new causes for corpus callosum development syndromes. Brain, 137, 1579-1613. doi:10.1093/brain/awt358 
Eliassen, J. C., Baynes, K., \& Gazzaniga, M. S. (2000). Anterior and posterior callosal contributions to simultaneous bimanual movements of the hands and fingers. Brain, $123 \mathrm{Pt}$ $12,2501-2511$.

Erickson, R. L., Paul, L. K., \& Brown, W. S. (2014). Verbal learning and memory in agenesis of the corpus callosum. Neuropsychologia, 60, 121-130. doi:10.1016/j.neuropsychologia.2014.06.003

Erickson, R. L., Young, C., Paul, L. K., \& Brown, W. S. (2013). WAIS-III index scores in individuals with agenesis of the corpus callosum [Abstract]. Presented at the Forty First Annual Meeting International Neuropsychological Society February 6-9, 2013 Waikoloa, Hawaii, USA. Journal of the International Neuropsychological Society, 19(S1), 224.

Fischer, M., Ryan, S. B., \& Dobyns, W. B. (1992). Mechanisms of interhemispheric transfer and patterns of cognitive function in acallosal patients of normal intelligence. Archives of Neurology, 49(3), 271-277.

Garrels, S. R., Paul, L. K., Schieffer, B. M., Florendo, E. V., Fox, M. M., Turk, A. A., \& Brown, W. S. (2001). Abstract problem solving in children with callosal agenesis [Abstract]. Presented at the Twenty-Ninth Annual International Neuropsychological Society Conference, February 14-17, 2001 Chicago, Illinois. Journal of the International Neuropsychological Society, 7(2), 258.

Geffen, G., Nilsson, J., Quinn, K., \& Teng, E. L. (1985). The effects of lesions of the corpus callosum on finger localization. Neuropsychologia, 23, 497-514.

Giedd, J. N., Castellanos, F. X., Casey, B. J., Kozuch, P., King, A. C., Hamburger, S. D., \& Rapoport, J. L. (1994). Quantitative morphology of the corpus callosum in attention deficit hyperactivity disorder. American Journal of Psychiatry, 151(5), 665-669. 
Glass, H., Shaw, G., Ma, C., \& Sherr, E. H. (2008). Agenesis of the corpus callosum in California 1983-2003: a population-based study. American Journal of Medical Genetics, 146A(19), 2495- 2500. doi:10.1002/ajmg.a.32418

Gooijers, J., Caeyenberghs, K., Sisti, H.M., Geurts, M., Heitger, M.H., Leemans, A., Swinnen, S.P. (2013) Diffusion tensor imaging metrics of the corpus callosum in relation to bimanual coordination: effect of task complexity and sensory feedback. Human Brain Mapping, 34(1), 241-245. doi: 10.1002/hbm.21429

Gott, P. S., \& Saul, R. E. (1978). Agenesis of the corpus callosum: limits of functional compensation. Neurology, 28(12), 1272-1279.

Guillem, P., Fabre, B., Cans, C., Robert-Gnansia, E., \& Jouk, P. S. (2003). Trends in elective terminations of pregnancy between 1989 and 2000 in a French county (the Isère). Prenatal Diagnosis, 23(11), 877-883. doi:10.1002/pd.711

Hanna, S. (2018). Academic functioning in individuals with agenesis of the corpus callosum (Unpublished doctoral dissertation). Fuller Graduate School of Psychology, Pasadena, California.

Imamura, T., Yamadori, A., Shiga, Y., Sahara, M., \& Abiko, H. (1994). Is disturbed transfer of learning in callosal agenesis due to a disconnection syndrome? Behavioural Neurology, 7, 43-48.

Jastak, S., \& Wilkinson, G. (1984). The Wide Range Achievement Test: Administration manual. Wilmington, DE: Jastak Association.

Jeeves, M. A. (1979). Some limits to interhemispheric integration in cases of callosal agenesis and partial commissurotomy. In I. S. Russell, M. W. Van Hof, \& G. Berlucchi (Eds.), Structure and function of the cerebral commissures (pp. 449 - 474). London: McMillan. 
Jeeves, M. A., \& Ettlinger, E. G. (1965). Psychological studies of the three cases of congenital agenesis of the corpus callosum Functions of the Corpus Callosum: CIBA Foundations Study Groups (Vol. 20, pp. 73 - 94).

Jeeves, M. A., \& Silver, P. H. (1988). Interhemispheric transfer of spatial tactile information in callosal agenesis and partial commissurotomy. Cortex, 24, 601-604.

Jeeves, M. A., Silver, P. H., \& Jacobson, I. (1988). Bimanual co-ordination in callosal agenesis and partial commissurotomy. Neuropsychologia, 26, 833-850.

Jeeves, M. A., Silver, P. H., \& Milner, A. D. (1988). Role of the corpus callosum in the development of a bimanual skills. Developmental Neuropsychology, 4, 305-323.

Kang, K. H., Paul, L. K., Castelli, F., \& Brown, W. S. (2009). Theory of mind in agenesis of the corpus callosum compared to high functioning autism [Abstract]. Poster presented at the Thirty-Seventh Annual Meeting International Neuropsychological Society: February 1114, 2009 Atlanta, Georgia, USA. Journal of the International Neuropsychological Society, 15(S1), 168. doi:10.1017/S1355617709090420

Kaplan, J. M., Brown, W. S., Adolphs, R., \& Paul, L. K. (2012). Psychological profile in agenesis of the corpus callosum [Abstract]. Poster presented at the Society for Neuroscience Annual Meeting: October 13- 17, 2012 New Orleans, LA, USA. 198.19.

Kourtidou, P., McCauley, S.R., Bigler, E.D., Traipe, E., Wu, T.C., Chu, Z.D., Hunter, J.V., Li, X., Levin, H.S., Wilde, E.A. (2013). Centrum Semiovale and Corpus Callosum Integrity in Relation to Information Processing Speed in Patients with Severe Traumatic Brain Injury. Journal of Head Trauma Rehabilitation, 28(6):433-41. DOI:10.1097/HTR.0b013e3182585d06

Koivisto, M. (2000). Interhemispheric interaction in semantic categorization of pictures. Cognitive Brain Research, 9(1), 45-51. PII: S0926-6410(99)00042-7. 
Lassonde, M., Sauerwein, H., Chicoine, A. J., \& Geoffroy, G. (1991). Absence of disconnexion syndrome in callosal agenesis and early callosotomy: brain reorganization or lack of structural specificity during ontogeny? Neuropsychologia, 29(6), 481-495.

Maertens, M., \& Pollmann, S. (2005). Interhemispheric resource sharing: Decreasing benefit with increasing processing efficiency. Brain and Cognition, 58(2), 183-192. doi:10.1016/j.bandc.2004.11.002.

Mangum, R. (2018). Self-understanding of executive function in individuals with agenesis of the corpus callosum. (Unpublished doctoral dissertation). Fuller Graduate School of Psychology, Pasadena, California.

Marco, E. J., Harrell, K. M., Brown, W. S., Hill, S. S., Jeremy, R. J., Kramer, J. H., . . Paul, L. K. (2012). Processing speed delays contribute to executive function deficits in individuals with agenesis of the corpus callosum. Journal of the International Neuropsychological Society, 18(3), 521-529. doi:10.1017/s1355617712000045.

Marion, S. D., Kilian, S. C., Naramor, T. L., \& Brown, W. S. (2003). Normal development of bimanual coordination: Visuomotor and interhemispheric contributions. Developmental Neuropsychology, 23(3), 399-421.doi:10.1207/s15326942dn2303_6

Mathias, J.L., Bigler, E.D., Jones , N.R., Bowden, S.C., Barrett-Woodbridge, M., Brown, G.C. \& Taylor, D.J. (2004) Neuropsychological and Information Processing Performance and Its Relationship to White Matter Changes Following Moderate and Severe Traumatic Brain Injury: A Preliminary Study, Applied Neuropsychology, 11:3, 134-152.

DOI:10.1207/s15324826an1103_2

Miller, J. S., Su, J. J., Paul, L. K., \& Brown, W. S. (2018). Adaptive behavior in agenesis of the corpus callosum: Self and informant reports. [Abstract]. Presented at the Forty First Annual Meeting International Neuropsychological Society February 14-17, 2018 
Washington, DC, USA. Journal of the International Neuropsychological Society, 24(S1), 195.

Mueller, K. L. O., Marion, S. D., Paul, L. K., \& Brown, W. S. (2009). Bimanual Motor Coordination in Agenesis of the Corpus Callosum. Behavioral Neuroscience, 123(5), 10001011. doi:10.1037/a0016868

Njiokiktjien, C., Ramaekers, G., \& Njiokiktjien, C. (1991). Absence of the corpus callosum: Clinicopathological correlations. Pediatric behavioural neurology: The child's corpus callosum (pp. 235 - 250).

Njiokiktjien, C., Valk, J., \& Ramaekers, G. (1988). Malformation or damage of the corpus callosum? A clinical and MRI study. Brain Development, 10(2), 92-99.

Norman, W., Jeeves, M.A., Milne, A., Ludwig, T. (1992) Hemispheric interactions: the bilateral advantage and task difficulty. Cortex. 28(4):623-42.

Paul, L. K., Brown, W. S., Adolphs, R., Tyszka, J. M., Richards, L. J., Mukherjee, P., \& Sherr, E. H. (2007). Agenesis of the corpus callosum: genetic, developmental and functional aspects of connectivity. Nature Reviews Neuroscience, 8(4), 287-299. doi:10.1038/nrn2107

Paul, L. K., Corsello, C., Kennedy, D. P., \& Adolphs, R. (2014). Agenesis of the corpus callosum and autism: a comprehensive comparison. Brain, 137, 1813-1829. doi:10.1093/brain/awu070

Paul, L. K., Erickson, R. L., Hartman, J. A., \& Brown, W. S. (2016). Learning and memory in individuals with agenesis of the corpus callosum. Neuropsychologia, 86, 183-192. doi:10.1016/j.neuropsychologia.2016.04.013

Paul, L. K., Lautzenhiser, A., Brown, W. S., Hart, A., Neumann, D., Spezio, M., \& Adolphs, R. (2006). Emotional arousal in agenesis of the corpus callosum. International Journal of Psychophysiology, 61(1), 47-56. doi:10.1016/j.jpsycho.2005.10.017 
Paul, L. K., Schieffer, B., \& Brown, W. S. (2004). Social processing deficits in agenesis of the corpus callosum: narratives from the Thematic Appreciation Test. Archives of Clinical Neuropsychology, 19(2), 215-225. doi:10.1016/S0887-6177(03)00024-6

Paul, L. K., Van Lancker-Sidtis, D., Schieffer, B., Dietrich, R., \& Brown, W. S. (2003). Communicative deficits in agenesis of the corpus callosum: nonliteral language and affective prosody. Brain and Language, 85(2), 313-324. doi:10.1016/s0093$934 x(03) 00062-2$

Pazienza, S. R., Brown, W. S., \& Paul, L. K. (2011). Emotional expressiveness and somatization in agenesis of the corpus callosum. [Abstract]. Poster presented at the Thirty-Ninth Annual Meeting International Neuropsychological Society: February 2-5, 2011 Boston, Massachusetts USA. Journal of the International Neuropsychological Society, 17(S1), 2. doi:10.1017/S1355617711000415.

Preilowski, B. F. (1972). Possible contribution of the anterior forebrain commissures to bilateral motor coordination. Neuropsychologia, 10(3), 267-277.

Raven, J. C. (1960). Guide to the Standard Progressive Matrices.

Raven, J. C. (1965). Guide to using the Colored Progressive Matrices.

Rehmel, J. L., Brown, W. S., \& Paul, L. K. (2016). Proverb comprehension in individuals with agenesis of the corpus callosum. Brain and Language, 160, 21-29. doi:10.1016/j.bandl.2016.07.001

Reuter-Lorenz, P. A., Stanczak, L., \& Miller, A. C. (1999). Neural recruitment and cognitive aging: Two hemispheres are better than one, especially as you age. Psychological Science, 10(6), 494-500. 
Sauerwein, H. C., \& Lassonde, M. (1994). Cognitive and sensori-motor functioning in the absence of the corpus callosum: neuropsychological studies in callosal agenesis and callosotomized patients. Behavioural Brain Research, 64(1-2), 229-240.

Sauerwein, H. C., Lassonde, M., Cardu, B., \& Geoffroy, G. (1981). Interhemispheric integration of sensory and motor functions in callosal agenesis. Neuropsychologia, 19, 445 - 454.

Saul, R. E., \& Sperry, R. W. (1968). Absence of commissurotomy symptoms with agenesis of the corpus callosum. Neurology, 18, 307.

Schell-Apacik, C. C., Wagner, K., Bihler, M., Ertl-Wagner, B., Heinrich, U., Klopocki, E., .. . von Voss, H. (2008). Agenesis and dysgenesis of the corpus callosum: clinical, genetic and neuroimaging findings in a series of 41 patients. American Journal of Medical Genetics, 146A(19), 2501- 2511. doi:10.1002/ajmg.a.32476

Schieffer, B., Paul, L. K., \& Brown, W. S. (2000). Deficits in complex concept formation in agenesis of the corpus callosum [Abstract]. Poster presented at the Twenty-Eighth Annual International Neuropsychological Society Conference: February 9-12, 2000 Denver, Colorado. Journal of the International Neuropsychological Society, 6(2), 164.

Schieffer, B., Paul, L. K., Schilmoeller, K., \& Brown, W. S. (2000). Components of intelligence and basic achievement in agenesis of the corpus callosum [Abstract]. Poster presented at the Twenty-Eighth Annual International Neuropsychological Society Conference: February 9-12, 2000 Denver, Colorado. Journal of the International Neuropsychological Society, 6(2), 164.

Solmaz, B., Tunc, B., Parker, D., Whyte, J., Hart, T., Rabinowitz, A., . . Verma, R. (2017). Assessing connectivity related injury burden in diffuse traumatic brain injury. Human Brain Mapping, 38(6), 2913-2922. doi:10.1002/hbm.23561 
Solursh, L. P., Margulies, A. I., Ashem, B., \& Stasiak, E. A. (1965). The relationships of agenesis of the corpus callosum to perception and learning. Journal of Nervous and Mental Disease, 141(2), 180-189.

Sperry, R. W. (1968). Hemisphere deconnection and unity in conscious awareness. American Psychologist, 23(10), 723-733.

Sperry, R. W., Gazzaniga, M., Bogen, J., Vinken, P. J., \& Bruyn, G. W. (1969). Interhemispheric relationships: the neocortical commissures; syndromes of hemisphere disconnection. Handbook of Clinical Neurology (Vol. 4, pp. 273-290).

Symington, S. H., Paul, L. K., Symington, M. F., Ono, M., \& Brown, W. S. (2010). Social cognition in individuals with agenesis of the corpus callosum. Social Neuroscience, 5(3), 296-308. doi:10.1080/17470910903462419

Tang, P. H., Bartha, A. I., Norton, M. E., Barkovich, A. J., Sherr, E. H., \& Glenn, O. A. (2009). Agenesis of the corpus callosum: an MR imaging analysis of associated abnormalities in the fetus. American Journal of Neuroradiology, 30(2), 257-263. doi:10.3174/ajnr.A1331

Turk, A., Brown, W. S., Symingtion, M., \& Paul, L. K. (2010). Social narratives in agenesis of the corpus callosum: linguistic analysis of the Thematic Apperception Test. Neuropsychologia, 48, 43-50. doi: 10.1016/j.neuropsychologia.2009.08.009

Ubukata, S., Ueda, K., Sugihara, G., Yassin, W., Aso, T., Fukuyama, H., \& Murai, T. (2016). Corpus callosum pathology as a potential surrogate marker of cognitive impairment in diffuse axonal injury. Journal of Neuropsychiatry and Clinical Neuroscience, 28(2), 97103. doi:10.1176/appi.neuropsych.15070159

Wahl, M., Strominger, Z., Jeremy, R. J., Barkovich, A. J., Wakahiro, I., Sherr, E. H., \& Mukherjee, P. (2009). Variability of homotopic and heterotopic callosal connectivity in partial agenesis of the corpus callosum: a 3T diffusion tensor imaging and q-ball 
tractography study. American Journal of Neuroradiology, 30(2), 282-289. doi:10.3174/ajnr.A1361

Wang, L. W., Huang, C. C., \& Yeh, T. F. (2004). Major brain lesions detected on sonographic screening of apparently normal term neonates. Neuroradiology, 46(5), 368-373. doi:10.1007/s00234-003-1160-4

Weissman, D. H., \& Banich, M. T. (2000). The cerebral hemispherescooperate to perform complex but not simple tasks. Neuropsychology, 14(1), 41-59.

Weissman, D. H., \& Compton, R. J. (2003). Practice makes a hemisphere perfect: The advantage of interhemispheric recruitment is eliminated with practice. Laterality: Asymmetries of Body, Brain, and Cognition, 8(4), 361-375.

Yakovlev, P. I., Lecours, A., \& Minkovski, A. (1967). The myelogenetic cycles of regional maturation of the brain. Regional Development of the Brain in Early Life (pp. 3-65).

Young, C. M., Erickson, R. L., Paul, L. K., \& Brown, W. S. (2013). Academic achievement in children and adults with agenesis of the corpus callosum. Poster presented at the Forty First Annual Meeting International Neuropsychological Society February 6-9, 2013 Waikoloa, Hawaii, USA. Journal of the International Neuropsychological Society, 19(S1), 44. doi:10.1017/S1355617713000362

Young, C. M., Folsom, R. C., Paul, L. K., Su, J., Mangum, R., \& Brown, W. S. (under review). Awareness of consequences in agenesis of the corpus callosum: semantic analysis of responses. 